Pacific Journal of Mathematic 


\title{
CONE RELATIONSHIPS OF BIORTHOGONAL SYSTEMS
}

\author{
S. W. SMITH
}

It is shown in this paper that total biorthogonal systems have the same cone if and only if they differ at most by rearrangement and by positive scalar multiplication. A connection is demonstrated between this result and work done by R. E. Fullerton in which he characterized the existence of an unconditional basis in terms of the existence of certain type cones. The paper is concluded by generalizing the first result to the situation in which two biorthogonal systems have cones which induce order isomorphic orderings.

1. Definitions and notations. In this paper we will assume that all vector spaces considered are real and that all topological vector spaces are Hausdorff. $E^{\prime}$ will denote the topological dual of $E$, and the letter $N$ will denote the set of natural numbers.

An ordered vector space is a vector space $E$ equipped with a transitive, reflexive relation $\leqq$ satisfying the following conditions:

(a) If $x, y, z$ are elements in $E$ and $x \leqq y$, then $x+z \leqq y+z$.

(b) If $x, y$ are elements in $E$ such that $x \leqq y$ and if $a$ is a nonnegative scalar, then $a x \leqq a y$.

An ordered topological vector space is a topological vector space which is also an ordered vector space. The positive cone $K$ of an ordered vector space $E$ is defined by $K=\{x \in E: x \geqq 0\}$. It has the properties that $K+K \subset K$ and $a K \subset K$ for each nonnegative scalar a. A subset $K$ of any vector space $E$ with these two properties is called a cone. If $K$ is a cone in the vector space $E$, then $(E, \leqq)$ is an ordered vector space where the relation $\leqq$ is defined on $E$ by $x \leqq y$ whenever $y-x \in K$.

If $x$ and $y$ are elements of $E$, then the order interval between $x$ and $y$ is the set $I(x, y)=\{z \in E: x \leqq z \leqq y\}$. The positive cone $K$ of $E$ is said to be generating if $E=K-K$ and proper if $K \cap-K=\{0\}$. If $E$ is an ordered topological vector space, its positive cone is said to be normal if there exists a local base of neighborhoods of zero for the given topology with the property that $V=\cup\{I(x, y\}: x, y \in V\}$ for each basic neighborhood $V$.

In a topological vector space $(E, T)$, a pair of indexed sets $\left(x_{\alpha}, f_{\alpha}\right)$, $\alpha \in A$, with $\left\{x_{\alpha}\right\} \subset E$ and $\left\{f_{\alpha}\right\} \subset E^{\prime}$ is called a biorthogonal system in $E$ if $f_{\alpha}\left(x_{\beta}\right)=0$ for $\alpha \neq \beta$ and $f_{\alpha}\left(x_{\alpha}\right)=1$. The set $K=\left\{x \in E: f_{\alpha}(x) \geqq\right.$ $0, \alpha \in A\}$ is a cone and is called the cone of the biorthogonal system $\left(x_{\alpha}, f_{\alpha}\right)$.

We will call a biorthogonal system $\left(x_{\alpha}, f_{\alpha}\right)$ total provided it has the following two properties: 
(1) The closed linear span of the $x_{\alpha}$ 's is $E$.

(2) The $f_{\alpha}$ 's are total, i.e., $f_{\alpha}(x)=0$ for each $\alpha \in A$ implies that $x=0$.

We will pay special attention to biorthogonal systems $\left(x_{n}, f_{n}\right), n \in N$, which are Schauder bases and will use the term basis to mean a Schauder basis.

2. Biorthogonal systems having the same cone. We will approach the proof of Theorem 2.6 by characterizing the extreme subsets of the positive cone. Propositions 2.4 and 2.5 although not used in the proof of Theorem 2.6, are of interest in themselves. An extreme subset of a cone $K$ is a convex set $A$ such that the following holds: if $u, v \in K, 0<t<1$, and $t u+(1-t) v \in A$, then $u$ and $v$ are elements of $A$.

If $x$ is a nonzero element of $K$, we will denote by $R(0, x)$ the ray $R(0, x)=\{t x: 0 \leqq t\}$. If $R(0, x)$ is an extreme subset of $K$, we will call it an extreme ray.

Proposition 2.1. Let $E$ be a vector space ordered by the cone $K$. Let $A$ be a convex subset of $K$. Then $A$ is an extreme subset of $K$ if and only if the following two conditions are satisfied:

(a) $I(0, x) \subset A$ for each $x \in A$.

(b) $R(0, x) \subset A$ for each $x \in A$.

Proof. Let $A$ be an extreme subset of $K$. Suppose that $A \neq \phi$ and that $A \neq\{0\}$. Let $x$ be a nonzero element of $A$, and let $\alpha>1$. Then $(\alpha-1) / \alpha \cdot 0+(1 / \alpha) \cdot(\alpha x)=x \in A$. Therefore, $0, \alpha x \in A$. Since $\alpha>1$ was arbitrary and $A$ is convex, $R(0, x) \subset A$. Now let $y \in I(0, x)$. Then $x=(1 / 2) \cdot 2(x-y)+(1 / 2) \cdot(2 y)$ and thus $2(x-y), 2 y \in A$. Therefore, $y \in A$ and hence $I(0, x) \subset A$.

Conversely, let $u, v \in K$ and $0<t<1$ such that $t u+(1-t) v=$ $x \in A$. Then $x-t u=(1-t) v \in K$. Therefore, $t u \in I(0, x)$ which implies that $t u \in A$ by (a) and hence that $u \in A$ by (b). Similarly $v$ is also an element of $A$. Therefore, $A$ is an extreme subset of $K$.

COROLLARY 2.2. Let $E$ be a vector space ordered by a cone $K$, and let $x$ be a nonzero elsment of $K$. Then the following statements are equivalent.

(a) $R(0, x)$ is an extreme ray

(b) $I(0, x) \subset R(0, x)$

(c) $I(0, x)=\{t x: 0 \in t \leqq 1\}$.

Proof. The implications $a \rightarrow b$ and $c \rightarrow a$ follow trivially from Proposition 2.1. To show that $b \rightarrow c$, we need only show that $I(0, x) \subset$ 
$\{t x: 0 \leqq t \leqq 1\}$. The reverse inclusion is clear. Thus suppose $z \in I(0, x)$. By (b) there exists $t \geqq 0$ such that $z=t x$. But $t x \in I(0, x)$ implies that $x-t x=(1-t) x \in I(0, x) \subset R(0, x)$. Then $1-t \geqq 0$ and $t \leqq 1$. Thus $z \in\{t x: 0 \leqq t \leqq 1\}$.

We remark that (c) of Corollary 2.2 is well known and is sometimes used as the definition of an extreme ray of a cone [5, p. 10].

Proposition 2.3. Let $E$ be a topological vector space ordered by the cone $K$ of a biorthogonal system $\left(x_{\alpha}, f_{\alpha}\right), \alpha \in A$, for which the $f_{\alpha}$ 's are total, and let $x \in E$. Then $R(0, x)$ is an extreme ray of $K$ if and only if there exists $\beta \in A$ and $b>0$ such that $x=b x_{\beta}$.

Proof. Suppose that $R(0, x)$ is an extreme ray for $K$. Since $x \neq 0$ and since the $f_{\alpha}$ 's are total, there exists $\beta \in A$ such that $f_{\beta}(x)>0$. Then $f_{\beta}(x) x_{\beta} \in I(0, x)$ which is contained in $R(0, x)$ by Corollary 2.2. Thus $R\left(0, x_{\beta}\right)=R(0, x)$ and there exists $b>0$ such that $x=b x_{\beta}$.

Conversely, suppose that $\alpha \in A$. Consider the ray $R\left(0, x_{\alpha}\right)$. If $z \in I\left(0, x_{\alpha}\right)$, then $f_{\beta}(z)=0$ for $\beta \neq \alpha$. Hence $f_{\beta}\left(z-f_{\alpha}(z) x_{\alpha}\right)=0$ for each $\beta \in A$. Since the $f_{\alpha}$ 's are total, we conclude that $z=f_{\alpha}(z) x_{\alpha}$. Thus $I\left(0, x_{\alpha}\right) \subset R\left(0, x_{\alpha}\right)$ and $R\left(0, x_{\alpha}\right)$ is an extreme ray, Corollary 2.2.

Proposition 2.4. If $E$ is a topological vector space ordered by the cone $K$ of biorthogonal system $\left(x_{\alpha}, f_{\alpha}\right)$, then $K$ has extreme rays if and only if the $f_{\alpha}^{\prime}$ 's are total.

Proof. If the $f_{\alpha}$ 's are total, then by Proposition 2.3, each ray $R\left(0, x_{\alpha}\right)$ is an extreme ray of $K$.

Suppose, however, that the $f_{\alpha}$ 's are not total. Then there exists a nonzero $x$ in $E$ such that $f_{\alpha}(x)=0$ for each $\alpha \in A$. Thus $x$ and $-x \in K$. If $y \neq 0$ is an element of $K$, then $(1 / 2) \cdot x+(1 / 2) \cdot-x=$ $0 \in R(0, y)$. However, either $x$ or $-x$ is not an element of $R(0, y)$, and thus $R(0, y)$ cannot be an extreme ray of $K$. Since $y$ was an arbitrary element of $K$, we conclude that $K$ has no extreme rays.

Proposition 2.5. Let $(E, T)$ be a topological vector space ordered by the cone $K$ of a biorthogonal system $\left(x_{n}, f_{n}\right)$ which is a basis on $K$, i.e., $x=\sum_{n=1}^{\infty} f_{n}(x) x_{n}$ for each $x \in K$. Then $A$ is a closed extreme subset of $K$ if and only if there exists a subset $\triangle$ of $N$ such that $A=$ $\left\{y \in K: f_{n}(y)=0\right.$ for $\left.n \in \Delta\right\}$.

Proof. Let $\Delta \subset N$ and let $A=\left\{y \in K: f_{n}(y)=0\right.$ for $\left.n \in \Delta\right\}$. It is clear that $I^{\prime}(0, x) \subset A$ and $R(0, x) \subset A$ for each $x \in A$. Therefore, by Proposition 2.1, $A$ is an extreme subset of $K$. Clearly $A$ is closed.

Conversely, suppose that $A$ is a closed extreme subset of $K$. Let 
$\Delta=\left\{n \in N: f_{n}(y)=0\right.$ for each $\left.y \in A\right\}$. We need only to show that $A=A_{\Delta}$ where $A_{\Delta}=\left\{y \in K: f_{n}(y)=0\right.$ for $\left.n \in \Delta\right\}$. Clearly $A \subset A_{\Delta}$. Thus suppose $z \in A_{\Delta}$, then $z=\sum_{n=1}^{\infty} f_{n}(z) x_{n}$ and $f_{n}(z)=0$ for $n \in \Delta$. Hence $z$ is contained in the closure of the convex hull of the $R\left(0, x_{n}\right), n \in N-\Delta$. However, for each $n \in N-\Delta$ there exists $y \in A$ such that $f_{n}(y)>0$. Since $f_{n}(y) x_{n}$ is an element of $I(0, y)$ and since $A$ is an extreme subset of $K$, we have that $R\left(0, f_{n}(y) x_{n}\right) \subset A$ by Proposition 2.1. Hence $R\left(0, x_{n}\right) \subset A$ for each $n \in N-\Delta . \quad A$ is also closed and convex, since it is a closed extreme subset of $K$. Therefore, $z \in A_{\Delta}$ and we have that $A=A_{\Delta}$.

THEOREM 2.6. Let $\left(x_{\alpha}, f_{\alpha}\right), \alpha \in A$, be a total biorthogonal system and $\left(y_{\beta}, h_{\beta}\right), \beta \in B$, a biorthogonal system with cones $K_{1}$ and $K_{2}$ respectively in a topological vector space $E$. Then $K_{1}=K_{2}$ if and only if there exists $a$ one-to-one mapping $F$ of $A$ onto $B$ and $a$ collection of positive scalars $\left\{\lambda_{\alpha}\right\}, \alpha \in A$, such that $y_{F^{\prime}(\alpha)}=\lambda_{\alpha} x_{\alpha}$ and $h_{F(\alpha)}=\left(1 / \lambda_{\alpha}\right) f_{\alpha}$.

Proof. If there exists a mapping $F$ and positive scalars $\left\{\lambda_{\alpha}\right\}, \alpha \in A$ as in the theorem statement, it is clear from the definitions of $K_{1}$ and $K_{2}$ that they must be equal.

Conversely, suppose that $K_{1}=K_{2}$. By Proposition 2.3, the collection of extreme rays of $K_{1}$ is the set $\left\{R\left(0, x_{\alpha}\right): \alpha \in A\right\}$. However, $K_{1} \cap-K_{1}=\{0\}$ since the $f_{\alpha}$ 's are total. Thus, because $K_{1}$ is also the cone of $\left(y_{\beta}, h_{\beta}\right)$, we must have the $h_{\beta}$ 's are also total. Applying Proposition 2.3 again, we have that the collection of extreme rays of $K_{1}$ is the set $\left\{R\left(0, y_{\beta}\right): \beta \in B\right\}$. Thus, $\left\{R\left(0, x_{\alpha}\right): \alpha \in A\right\}=\left\{R\left(0, y_{\beta}\right): \beta \in B\right\}$. Define $F$ mapping $A$ into $B$ in the following way: $\alpha$ maps to $F(\alpha)$ provided $R\left(0, x_{\alpha}\right)=R\left(0, y_{\left.F^{(} \alpha\right)}\right)$. It is a simple matter to verify that $F$ is a well-defined, one-to-one mapping of $A$ onto $B$. Therefore, for each $\alpha \in A$ there exists $\lambda_{\alpha}>0$ such that $y_{F^{\prime}(\alpha)}=\lambda_{\alpha} x_{\alpha}$. Furthermore, since the closed linear span of the $x_{\alpha}$ 's is $E, h_{F^{(\alpha)}}=\left(1 / \lambda_{\alpha}\right) f_{\alpha}$, [3, Proposition 1].

Corollary 2.7. Let $(E, T)$ be a topological vector space ordered by the cone $K$ of basis $\left(x_{n}, f_{n}\right)$. If $\left(y_{\alpha}, h_{\alpha}\right), \alpha \in A$, is any biorthogonal system whose cone is also $K$, then $A=N$ and there exists a permutation $\tau$ of $N$ such that $\left(y_{\tau(n)}, h_{\tau(n)}\right)$ is also a basis.

Proof. By Theorem 2.6, there exists a one-to-one mapping of $N$ onto $A$ and positive scalars $\left\{b_{n}: n \in N\right\}$ such that $y_{\tau(n)}=b_{n} x_{n}$ and $h_{\tau(n)}=\left(1 / b_{n}\right) f_{n}$. Since $\left(x_{n}, f_{n}\right)$ is a basis, $\left(b_{n} x_{n},\left(1 / b_{n}\right) f_{n}\right)=\left(y_{\tau(n)}, h_{\tau(n)}\right)$ is also a basis.

COROLlary 2.8. If $(E, T)$ is a topological vector space ordered by the cone $K$ of an unconditional basis $\left(x_{n}, f_{n}\right)$ and if $\left(y_{\alpha}, h_{\alpha}\right), \alpha \in A$, is 
any biorthogonal system having the same cone $K$, then $A=N$ and $\left(y_{n}, h_{n}\right)$ is also an unconditional basis $(E, T)$.

3. Remarks. We next point out a connection between the above work and some work done by Fullerton [4]. To do this let us notice that if $\left(x_{n}, f_{n}\right)$ is a basis in a locally convex topological vector space $(E, T)$, then the rays $\left\{R\left(0, x_{n}\right): n \in N\right\}$ satisfy the following conditions.

(a) $\bigcup\left\{R\left(0, x_{n}\right): n \in N\right\}$ generates a linear space dense in $E$.

(b) For each $n \in N$, the closed linear subspace $L_{n}$ generated by the set $\left\{R\left(0, x_{j}\right): j \neq n\right\}$ is a hyperplane.

(c) $\bigcap\left\{L_{n}: n \in N\right\}=\{0\}$.

Furthermore, if $H_{n}$ is the closed half-space bounded by $L_{n}$ and containing $R\left(0, x_{n}\right)$, then $K=\bigcap\left\{H_{n}: n \in N\right\}$ is a cone and in fact is the cone of the basis $\left(x_{n}, f_{n}\right)$.

If $\left(x_{n}, f_{n}\right)$ is an unconditional basis and $(E, T)$ is sequentially complete, it is true that the cone $K$ generated by the rays $\left\{R\left(0, x_{n}\right): n \in N\right\}$ satisfies two additional conditions.

(d) $K \cap x-K$ is compact for each $x \in K$.

(e) $K-K=E$.

Statement (d) is equivalent to unconditional convergence of the series $\sum_{n=1}^{\infty} f_{n}(x) x_{n}$ for each $x \in K[9, \mathrm{p} .16]$. Statement (e) is well known and follows from the fact that in a sequentially complete space unconditional convergence is equivalent to sub-series convergence [8, p. 17].

REMARK. In a complete locally convex topological vector space, the existence of an unconditional basis is equivalent to the existence of a cone $K$ defined by a collection of rays $\left\{R\left(0, x_{n}\right): n \in N\right\}$ satisfying conditions (a) through (e). Furthermore, it can be shown that $K$ is the cone of that basis.

The above remark is essentially the work of Fullerton [4]; however, it can also be obtained using a theorem of McArthur [9, p. 16].

We would like to point out here that even though Fullerton did not include condition (e) when claiming the above remark to be true, it is necessary. This can be seen by the following example. Let $(E, T)=C[0,1]$ with the sup-norm topology. Let $\left(x_{n}, f_{n}\right)$ be the usual Schauder basis given for $C[0,1],[2, \mathrm{p} .69]$. It is well known that the cone $K$ of this basis is normal. Thus by theorems of McArthur [10, pp. 6 and 16], we have that $K \cap x-K$ is compact for each $x \in K$. Consequently, the rays $R\left(0, x_{n}\right)$ of this basis satisfy conditions (a) through (d) and the cone $K$ would be called an absolute basis cone by Fullerton [4]. Hence, by Fullerton's statement of the above remark, the existence of the cone $K$ is equivalent to the existence of an un- 
conditional basis system $\left(y_{\alpha}, h_{\alpha}\right), \alpha \in A$, having $K$ as its cone. It is well known that the basis $\left(x_{n}, f_{n}\right)$ above is a conditional basis [7]. Furthermore by Theorem 2.6, if $\left(y_{\alpha}, h_{\alpha}\right), \alpha \in A$, is any arbitrary biorthogonal system having $K$ for a cone, then $A=N$ and there exists a permutation $\tau$ of $N$ and a collection $\left\{\lambda_{n}: n \in N\right\}$ of positive scalars such that $y_{n}=\lambda_{n} x_{\tau(n)}$. Since $\left(x_{n}, f_{n}\right)$ is a conditional basis, $\left(\lambda_{n} x_{\tau(n)}\right.$, $\left.\left(1 / \lambda_{n}\right) f_{\tau(n)}\right)$ must also be a conditional basis if indeed it is a basis at all. Thus we have that $K$ satisfied conditions (a) through (d), but no unconditional basis system exists which has $K$ for its cone. We note that $K$ is not generating since it is the cone of a conditional basis and is normal [9, p. 20]. Hence, condition (e) is indeed necessary in order that the above remark be true.

Note that Corollary 2.8 states that unconditional basis cones are unique among biorthogonal system cones in any topological vector space. Therefore, it seems likely that the above remark could be generalized to general topological vector spaces. Corollary 2.7 indicates that some type of a similar remark should be true for conditional bases.

4. Biorthogonal systems giving order isomorphic orderings. In this section we will prove a type of analogue to Theorem 2.6. For this work one might think of two cones as being the same if they give order isomorphic orderings.

If $\left(E_{1}, T_{1}, K_{1}\right)$ and $\left(E_{2}, T_{2}, K_{2}\right)$ are ordered topological vector spaces with positive cones $K_{1}$ and $K_{2}$ respectively, then $E_{1}$ and $E_{2}$ are said to be order isomorphic to each other if there exist a linear isomorphism $T$ mapping $E_{1}$ onto $E_{2}$ such that $T\left(K_{1}\right)=K_{2}$. If $T$ and $T^{-1}$ are also continuous, $E_{1}$ and $E_{2}$ are topologically order isomorphic.

Let $\left(x_{\alpha}, f_{\alpha}\right)$ be a biorthogonal system in a topological vector space $E_{1}$ and $\left(y_{\alpha}, h_{\alpha}\right)$ a biorthogonal system in a topological vector space $E_{2}$. We say that $\left(x_{\alpha}, f_{\alpha}\right)$ and $\left(y_{\alpha}, h_{\alpha}\right)$ are equivalent systems if for each $x \in E_{1}$ and $y \in E_{2}$ there exists a corresponding $y^{\prime} \in E_{2}$ and $x^{\prime} \in E_{1}$ such that $f_{\alpha}(x)=h_{\alpha}\left(y^{\prime}\right)$ and $f_{\alpha}\left(x^{\prime}\right)=h_{\alpha}(y)$ for each $\alpha$. If $\left(x_{n}, f_{n}\right)$ and $\left(y_{n}, h_{n}\right)$ are Schauder bases for $E_{1}$ and $E_{2}$ respectively, we say that $\left(x_{n}, f_{n}\right)$ and $\left(y_{n}, h_{n}\right)$ are equivalent bases provided $\left\{\left(a_{n}\right): \sum_{n=1}^{\infty} a_{n} x_{n}\right.$ converges in $\left.E_{1}\right\}=$ $\left\{\left(b_{n}\right): \sum_{n=1}^{\infty} b_{n} y_{n}\right.$ converges in $\left.E_{2}\right\}$.

THEOREM 4.1. Let $\left(E_{1}, K_{1}\right)$ and $\left(E_{2}, K_{2}\right)$ be topological vector spaces ordered respectively by the cones $K_{1}$ and $K_{2}$ of total biorthogonal systems $\left(x_{\alpha}, f_{\alpha}\right)$ and $\left(y_{\alpha}, h_{\alpha}\right), \alpha \in A$. The following statements can be proved.

(a) If $\left(E_{1}, K_{1}\right)$ and $\left(E_{2}, K_{2}\right)$ are topologically order isomorphic, there exists a permutation $\tau$ of $A$ and positive scalars $\left\{\lambda_{\alpha}: \alpha \in A\right\}$ such that $\left(x_{\alpha}, f_{\alpha}\right)$ and $\left(\lambda_{\alpha} y_{\tau(\alpha)},\left(1 / \lambda_{\alpha}\right) h_{\tau(\alpha)}\right)$ are equivalent systems.

(b) If $\left(x_{\alpha}, f_{\alpha}\right)$ and $\left(y_{\alpha}, h_{\alpha}\right)$ are equivalent, then $\left(E_{1}, K_{1}\right)$ and $\left(E_{2}, K_{2}\right)$ 
are order isomorphic vector spaces.

Proof. Let $T: E_{1} \rightarrow E_{2}$ be a topological order isomorphism mapping $E_{1}$ onto $E_{2}$. Then $\left(T\left(x_{\alpha}\right), f_{\alpha} T^{-1}\right)$ is a biorthogonal system in $E_{2}$. Let $K_{3}$ denote the cone of $\left(T\left(x_{\alpha}\right), f_{\alpha} T^{-1}\right)$. Then $K_{2}=K_{3}$. Therefore by Theorem 2.6, there exists a permutation of $A$ and positive scalars $\left\{\lambda_{\alpha}: \alpha \in A\right\}$ such that $T\left(x_{\alpha}\right)=\lambda_{\alpha} y_{\tau(\alpha)}$ and $f_{\alpha} T^{-1}=\left(1 / \lambda_{\alpha}\right) h_{\tau(\alpha)}$. Furthermore $\left(x_{\alpha}, f_{\alpha}\right)$ and $\left(\lambda_{\alpha} y_{\tau(\alpha)},\left(1 / \lambda_{\alpha}\right) h_{\tau(\alpha)}\right)$ are equivalent systems.

Suppose now that $\left(x_{\alpha}, f_{\alpha}\right)$ and $\left(y_{\alpha}, h_{\alpha}\right)$ are equivalent. If $x \in E_{1}$, let $T(x)$ denote that element $y$ of $E_{2}$ for which $f_{\alpha}(x)=h_{\alpha}(y)$ for each $\alpha \in A$. Then $T$ is a linear isomorphism of $E_{1}$ onto $E_{2}$ and $T\left(K_{1}\right)=K_{2}$.

Corollary 4.2. Let $(E, T)$ be a sequentially complete topological vector space ordered by the cone $K_{1}$ of an unconditional basis $\left(x_{n}, f_{n}\right)$. Let $\left\{b_{n}: n \in N\right\}$ be any sequence of nonzero scalars, $z_{n}=b_{n} x_{n}$, and $h_{n}=$ $\left(1 / b_{n}\right) f_{n}$. Then if $K_{2}$ is the cone of $\left(z_{n}, h_{n}\right)$, we have that $\left(E, K_{1}\right)$ and $\left(E, K_{2}\right)$ are order isomorphic as vector spaces.

Proof. We begin by showing that $\left(x_{n}, f_{n}\right)$ and $\left(a_{n} x_{n}, a_{n} f_{n}\right)$ are equivalent bases where $a_{n}=\operatorname{sgn} b_{n}$. In a sequentially complete space, $\sum_{n=1}^{\infty} f_{n}(x) x_{n}$ converges unconditionally to $x$ if and only if $\sum_{n=1}^{\infty} d_{n} f_{n}(x) x_{n}$ converges to $x$ for every sequence $\left\{d_{n}: d_{n}= \pm 1\right\},[8, \mathrm{p} .17]$. Using this fact again we have that $\sum_{n=1}^{\infty} d_{n} f_{n}(x) x_{n}$ converges unconditionally to $x$ for each such sequence and for each $x \in E$. Thus $\sum_{n=1}^{\infty} b_{n} x_{n}$ converges if and only if $\sum_{n=1}^{\infty} b_{n}\left(a_{n} x_{n}\right)$ converges, i.e., $\left(x_{n}, f_{n}\right)$ and $\left(a_{n} x_{n}, a_{n} f_{n}\right)$ are equivalent bases. Thus by (b) of Theorem 4.1 , we have that $\left(E, K_{1}\right)$ and $\left(E, K_{3}\right)$ are order isomorphic vector spaces where $K_{3}$ is the cone of the basis $\left(a_{n} x_{n}, a_{n} f_{n}\right)$. However, $K_{2}=K_{3}$, thus $\left(E, K_{1}\right)$ and $\left(E, K_{2}\right)$ are order isomorphic vector spaces.

Proposition 4.3. Let $E_{1}$ and $E_{2}$ be complete metric linear spaces ordered by the cones $K_{1}$ and $K_{2}$ respectively of the total biorthogonal systems $\left(x_{\alpha}, f_{\alpha}\right)$ and $\left(y_{\alpha}, h_{\alpha}\right), \alpha \in A$. Then $\left(E_{1}, K_{1}\right)$ and $\left(E_{2}, K_{2}\right)$ are topologically order isomorphic if and only if there exists positive scalars $\left\{\lambda_{\alpha}: \alpha \in A\right\}$ and a permutation $\tau$ of $A$ such that the systems $\left(x_{\alpha}, f_{\alpha}\right), \alpha \in A$, and $\left(\lambda_{\alpha} y_{\tau(\alpha)},\left(1 / \lambda_{\alpha}\right) h_{\tau(\alpha)}\right)$ are equivalent.

Proof. If $\left(E_{1}, K_{1}\right)$ and $\left(E_{2}, K_{2}\right)$ are order isomorphic topological vector spaces, we get the desired result by applying (a) of Theorem 4.1.

Conversely, we can assume without loss of generality that $\left(x_{\alpha}, f_{\alpha}\right)$ and $\left(y_{\alpha}, h_{\alpha}\right)$ are equivalent. Then by the isomorphism theorem of Arsove and Edwards [1], there exists an isomorphism $T$ of $E_{1}$ onto $E_{2}$ such that $T\left(x_{\alpha}\right)=y_{\alpha}$. Furthermore $T$ is defined as in the proof 
of (b) of Theorem 2.6. Hence $T\left(K_{1}\right)=K_{2}$, i.e., $T$ is a topological order isomorphism.

Proposition 4.4. Let $\left(E_{1}, T_{1}\right)$ and $\left(E_{2}, T_{2}\right)$ be barrelled spaces ordered by the cones $K_{1}$ and $K_{2}$ of Schauder bases $\left(x_{n}, f_{n}\right)$ and $\left(y_{n}, h_{n}\right)$ respectively. Then $\left(E_{1}, T_{1}, K_{1}\right)$ and $\left(E_{2}, T_{2}, K_{2}\right)$ are topologically order isomorphic if and only if there exists a permutation $\tau$ of $N$ and positive scalars $\left\{\lambda_{n}: n \in N\right\}$ such that $\left(x_{n}, f_{n}\right)$ and $\left(\lambda_{n} y_{\tau(n)},\left(1 / \lambda_{n}\right) h_{\tau(n)}\right)$ are equivalent bases.

Proof. Suppose that $\left(E_{1}, T_{1}, K_{1}\right)$ and $\left(E_{2}, T_{2}, K_{2}\right)$ are topologically order isomorphic. Let $T: E_{1} \rightarrow E_{2}$ be the defining order homeomorphism. As in the proof of Theorem 4.1, there exists a permutation $\tau$ of $N$ and positive scalars $\left\{\lambda_{n}: n \in N\right\}$ such that $T\left(x_{n}\right)=\lambda_{n} y_{\tau(n)}$. Thus $\left(x_{n}, f_{n}\right)$ and $\left(\lambda_{n} y_{\tau(n)},\left(1 / \lambda_{n}\right) h_{\tau(n)}\right)$ are equivalent bases [6, p. 678].

Conversely, we can assume without loss of generality that $\left(x_{n}, f_{n}\right)$ and $\left(y_{n}, h_{n}\right)$ are equivalent bases. Then $F: E_{1} \rightarrow E_{2}$ defined by $F(x)=$ $\sum_{n=1}^{\infty} f_{n}(x) y_{n}$ is a linear homeomorphism [6, p. 678] and clearly $F\left(K_{1}\right)=K_{2}$.

\section{BIBLIOGRAPHY}

1. M. G. Arsove and R. E. Edwards, Generalized bases in topological linear spaces, Studia Math. 19 (1960), 95-113.

2. M. M. Day, Normed Linear Spaces, 2nd ed., Academic Press, New York, 1962.

3. J. Dieudonne, On biorthogonal systems, Michigan Math. J. 2 (1954), 7-20.

4. R. E. Fullerton, Geometric structure of absolute basis systems in a linear topological space, Pacific J. Math. 12 (1962), 137-147.

5. G. J. O. Jameson, Ordered linear spaces (submitted for publication)

6. O. T. Jones and J. R. Retherford, On similar bases in barrelled spaces, Proc. Amer. Math. Soc. 18 (1967), 677-680.

7. S. Karlin, Bases in Banach spaces, Duke Math. J. 15 (1948), 971-985.

8. C. W. McArthur, In finite series, Lecture notes, Florida State Univ., Tallahassee, Florida, 1967.

9.___ Ordered vector spaces, Lecture notes, Florida State Univ., Tallahassee, Florida, 1968.

10. Convergence of monotone nets in ordered topological vector spaces (submitted for publication)

Received March 26, 1970. The material in this paper is part of the author's Doctoral Dissertation written at Florida State University under the guidance of Professor C. W. McArthur.

\section{TeXas Tech UnIVERsity}




\section{PACIFIC JOURNAL OF MATHEMATICS}

\section{EDITORS}

\author{
H. SAMELSON \\ Stanford University \\ Stanford, California 94305 \\ Richard Pierce \\ University of Washington \\ Seattle, Washington 98105
}

\author{
J. DugundJI \\ Department of Mathematics \\ University of Southern California \\ Los Angeles, California 90007 \\ RICHARD ARENS \\ University of California \\ Los Angeles, California 9.0024
}

\section{ASSOCIATE EDITORS}

\begin{tabular}{|c|c|}
\hline E. F. BECKENBACH & K. YoshidA \\
\hline \multicolumn{2}{|c|}{ SUPPORTING INSTITUTIONS } \\
\hline UNIVERSITY OF BRITISH COLUMBIA & STANFORD UNIVERSITY \\
\hline CALIFORNIA INSTITUTE OF TECHNOLOGY & UNIVERSITY OF TOKYO \\
\hline UNIVERSITY OF CALIFORNIA & UNIVERSITY OF UTAH \\
\hline MONTANA STATE UNIVERSITY & WASHINGTON STATE UNIVERSITY \\
\hline UNIVERSITY OF NEVADA & UNIVERSITY OF WASHINGTON \\
\hline NEW MEXICO STATE UNIVERSITY & $* \quad * \quad *$ \\
\hline OREGON STATE UNIVERSITY & AMERICAN MATHEMATICAL SOCIETY \\
\hline UNIVERSITY OF OREGON & CHEVRON RESEARCH CORPORATION \\
\hline OSAKA UNIVERSITY & TRW SYSTEMS \\
\hline UNIVERSITY OF SOUTHERN CALIFORNIA & NAVAL WEAPONS CENTER \\
\hline
\end{tabular}

The Supporting Institutions listed above contribute to the cost of publication of this Journal, but they are not owners or publishers and have no responsibility for its content or policies.

Mathematical papers intended for publication in the Pacific Journal of Mathematics should be in typed form or offset-reproduced, (not dittoed), double spaced with large margins. Underline Greek letters in red, German in green, and script in blue. The first paragraph or two must be capable of being used separately as a synopsis of the entire paper. The editorial "we" must not be used in the synopsis, and items of the bibliography should not be cited there unless absolutely necessary, in which case they must be identified by author and Journal, rather than by item number. Manuscripts, in duplicate if possible, may be sent to any one of the four editors. Please classify according to the scheme of Math. Rev. Index to Vol. 39. All other communications to the editors should be addressed to the managing editor, Richard Arens, University of California, Los Angeles, California, 90024.

50 reprints are provided free for each article; additional copies may be obtained at cost in multiples of 50 .

The Pacific Journal of Mathematics is published monthly. Effective with Volume 16 the price per volume (3 numbers) is $\$ 8.00$; single issues, $\$ 3.00$. Special price for current issues to individual faculty members of supporting institutions and to individual members of the American Mathematical Society: $\$ 4.00$ per volume; single issues $\$ 1.50$. Back numbers are available.

Subscriptions, orders for back numbers, and changes of address should be sent to Pacific Journal of Mathematics, 103 Highland Boulevard, Berkeley, California, 94708.

PUBLISHED BY PACIFIC JOURNAL OF MATHEMATICS, A NON-PROFIT CORPORATION

Printed at Kokusai Bunken Insatsusha (Internatıonal Academic Printing Co., Ltd.), 7-17, Fujimi 2-chome, Chiyoda-ku, Tokyo, Japan. 


\section{Pacific Journal of Mathematics}

\section{Vol. 35, No. $3 \quad$ November, 1970}

John D. Arrison and Michael Rich, On nearly commutative degree one algebras . . . 533

Bruce Alan Barnes, Algebras with minimal left ideals which are Hilbert spaces . . . . 537

Robert F. Brown, An elementary proof of the uniqueness of the fixed point index . . . 549

Ronn L. Carpenter, Principal ideals in F-algebras .................... 559

Chen Chung Chang and Yiannis (John) Nicolas Moschovakis, The Suslin-Kleene

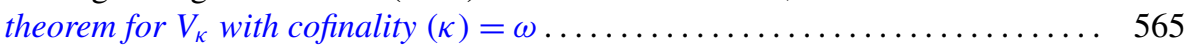

Theodore Seio Chihara, The derived set of the spectrum of a distribution

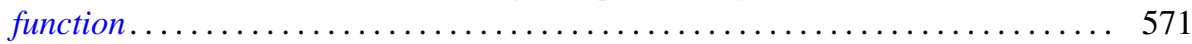

Tae Geun Cho, On the Choquet boundary for a nonclosed subspace of $C(S) \ldots \ldots \quad 575$

Richard Brian Darst, The Lebesgue decomposition, Radon-Nikodym derivative,

conditional expectation, and martingale convergence for lattices of sets .......

David E. Fields, Dimension theory in power series rings . . . . . . . . . . . .

Michael Lawrence Fredman, Congruence formulas obtained by counting

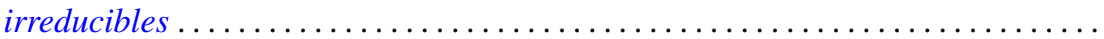

John Eric Gilbert, On the ideal structure of some algebras of analytic functions.....

G. Goss and Giovanni Viglino, Some topological properties weaker than

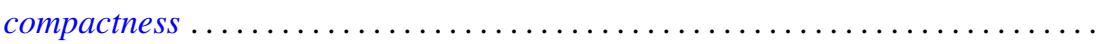

581

601

625

George Grätzer and J. Sichler, On the endomorphism semigroup (and category) of

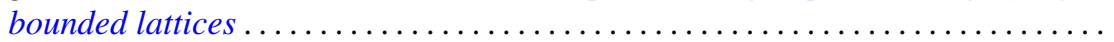

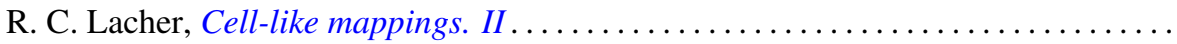

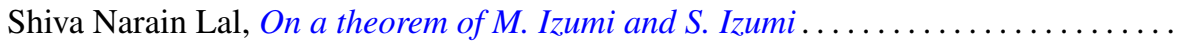

661

Howard Barrow Lambert, Differential mappings on a vector space ...............

Richard G. Levin and Takayuki Tamura, Notes on commutative power joined

semigroups.

Robert Edward Lewand and Kevin Mor McCrimmon, Macdonald's theorem for quadratic Jordan algebras.

J. A. Marti, On some types of completeness in topological vector spaces ....

Walter J. Meyer, Characterization of the Steiner point

717

Saad H. Mohamed, Rings whose homomorphic images are $q$-rings ...

727

Thomas V. O'Brien and William Lawrence Reddy, Each compact orientable surface

of positive genus admits an expansive homeomorphism ...

737

Robert James Plemmons and M. T. West, On the semigroup of binary relations...

743

Calvin R. Putnam, Unbounded inverses of hyponormal operator . .

755

William T. Reid, Some remarks on special disconjugacy criteria for differential

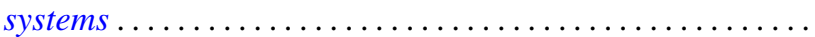

C. Ambrose Rogers, The convex generation of convex Borel sets in euclidean

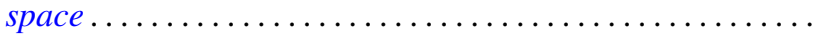

S. Saran, A general theorem for bilinear generating functions .

S. W. Smith, Cone relationships of biorthogonal systems ......

Wolmer Vasconcelos, On commutative endomorphism rings ....

795

Vernon Emil Zander, Products of finitely additive set functions from Orlicz

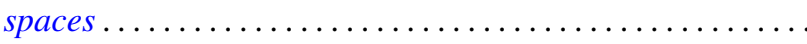

G. Sankaranarayanan and C. Suyambulingom, Correction to: "Some renewal

theorems concerning a sequence of correlated random variables" .

Joseph Zaks, Correction to: "Trivially extending decompositions of $E^{n}$ ”....... 805

Dong Hoon Lee, Correction to: "The adjoint group of Lie groups" ............ 805

James Edward Ward, Correction to: "Two-groups and Jordan algebras". 